\title{
Immunogenicity of native and CD4 liganded monomeric and trimeric envelope glycoproteins based on HIV-1 Subtype C consensus Founder virus sequences
}

\author{
MA Killick*, A Capovilla, MA Papathanasopoulos
}

From AIDS Vaccine 2012

Boston, MA, USA. 9-12 September 2012

\section{Background}

The ability to induce a broadly neutralizing antibody (bNAb) response following vaccination is regarded as a crucial aspect in developing an effective HIV-1 vaccine. This study describes the design and construction of a subtype $C$ founder virus consensus Env immunogen derived from newly transmitted/founder virus sequences, and its immunogenicity testing in the presence or absence of liganded CD4, in small animals.

\section{Methods}

Monomeric (gp120), dimeric (gp120GCN4) and trimeric (gp140GCN4 +/-) founder virus conformations were expressed in mammalian cell culture. Unliganded or $2 \mathrm{dCD} 4^{\mathrm{S} 60 \mathrm{C}}$ liganded Env glycoproteins were purified by lectin affinity chromatography, followed by conformation and complex purification using size exclusion chromatography. Immunogens/immune complexes were evaluated by ELISA, SDS-PAGE, Native PAGE and Surface Plasmon Resonance. Immunogenicity of each conformation alone or complexed to $2 \mathrm{dCD} 4^{\mathrm{S} 60 \mathrm{C}}$ was evaluated in rabbits. Breadth and potency of the rabbit sera was tested against 12 pseudoviruses (Tiers 1-3), derived from HIV-1 subtype B and C Env, using the PhenoSense Neutralizing antibody assay (Monogram Bioscience Inc.).

\section{Results}

Minimal neutralizing breadth was obtained from animals immunized exclusively with Env conformations. However, animals that received the Env/2dCD $4^{\mathrm{S} 60 \mathrm{C}}$ complex showed extensive neutralizing capacity against all 12 viruses tested, including the tier 2 and 3 virus strains. End-point ELISA titre results revealed that the rabbits that were immunized with Env/2dCD $4^{\mathrm{S} 60 \mathrm{C}}$ produced both Env and $2 \mathrm{dCD} 4$ specific titres, but those directed towards $2 \mathrm{dCD} 4$ were on average $10 \mathrm{x}$ lower than the $2 \mathrm{dCD} 4$ control group. This implies a proportion of the neutralizing antibody activity is directed towards conserved epitopes exposed on the Env/ $2 \mathrm{dCD} 4^{\mathrm{S} 60 \mathrm{C}}$ immunogens.

\section{Conclusion}

The ability to induce bNAb activity in previous immunization studies utilizing Env/CD4 complexes was attributed to the induction of high anti-CD4 titres. By contrast, in our study the relatively low anti-CD4 titres compared to anti-Env titres and neutralization profiles suggest an alternative mechanism of neutralization other than a response directed to $\mathrm{CD} 4$ alone.

Published: 13 September 2012

\section{doi:10.1186/1742-4690-9-S2-P21}

Cite this article as: Killick et al:: Immunogenicity of native and CD4 liganded monomeric and trimeric envelope glycoproteins based on HIV-1 Subtype C consensus Founder virus sequences. Retrovirology 2012 9(Suppl 2):P21.

HIV Pathogenesis Research Laboratory, Johannesburg, South Africa

( 2012 Killick et al; licensee BioMed Central Ltd. This is an Open Access article distributed under the terms of the Creative Commons 